\title{
SUSTAINABILITY EFFECTS OF NEXT-GENERATION INTERSECTION CONTROL FOR AUTONOMOUS VEHICLES
}

\author{
Zhixia (Richard) $\mathrm{Li}^{1^{*}}$, Madhav V. Chitturi ${ }^{2 * *}$, Lang $\mathrm{Yu}^{3}$, Andrea R. Bill ${ }^{4}$, David A. Noyce ${ }^{5}$ \\ ${ }^{1}$ Dept of Civil and Environmental Engineering, University of Louisville, United States \\ 2,3,4,5 Traffic Operations and Safety (TOPS) Laboratory, Dept of Civil and Environmental Engineering, \\ University of Wisconsin-Madison, Madison, Wisconsin, United States
}

Submitted 31 December 2014; resubmitted 12 April 2015; accepted 17 April 2015

\begin{abstract}
Transportation sustainability is adversely affected by recurring traffic congestions, especially at urban intersections. Frequent vehicle deceleration and acceleration caused by stop-and-go behaviours at intersections due to congestion adversely impacts energy consumption and ambient air quality. Availability of the maturing vehicle technologies such as autonomous vehicles and Vehicle-To-Vehicle (V2V) / Vehicle-To-Infrastructure (V2I) communications provides technical feasibility to develop solutions that can reduce vehicle stops at intersections, hence enhance the sustainability of intersections. This paper presents a next-generation intersection control system for autonomous vehicles, which is named ACUTA. ACUTA employs an enhanced reservation-based control algorithm that controls autonomous vehicles' passing sequence at an intersection. Particularly, the intersection is divided into $n$-by- $n$ tiles. An intersection controller reserves certain time-space for each vehicle, and assures no conflict exists between reservations. The algorithm was modelled in microscopic traffic simulation platform VISSIM. ACUTA algorithm modelling as well as enhancement strategies to minimize vehicle intersection stops and eventually emission and energy consumption were discussed in the paper. Sustainability benefits offered by this next-generation intersection were evaluated and compared with traditional intersection control strategies. The evaluation reveals that multi-tile ACUTA reduces carbon monoxide (CO) and Particulate Matter (PM) 2.5 emissions by about 5\% under low to moderate volume conditions and by about $3 \%$ under high volume condition. Meanwhile, energy consumption is reduced by about $4 \%$ under low to moderate volume conditions and by about $12 \%$ under high volume condition. Compared with four-way stop control, single-tile ACUTA reduces CO and PM 2.5 emissions as well as energy consumption by about $15 \%$ under any prevailing volume conditions. These findings validated the sustainability benefits of employing next-generation vehicle technologies in intersection traffic control. In addition, extending the ACUTA to corridor level was explored in the paper.
\end{abstract}

Keywords: vehicle emission; sustainability; autonomous vehicles; V2I communications; traffic control; connected vehicles; energy consumption; intersection.

\section{Introduction}

Traffic congestion adversely affects transportation mobility, energy consumption, and ambient air quality, which are regarded as a major cause that restrains the sustainability of transportation network. With consideration of the increasing traffic demand, United States (US) Federal Highway Administration (FHWA) estimates that by 2020, 29\% of urban National Highway System (NHS) routes in the US will be congested for much of the day, and $42 \%$ of NHS routes will be congested during peak periods (CamSys 2005). As a result of congestion, trans- portation related emissions already accounted for $82 \%$ of carbon monoxide (CO) and 56\% of nitrogen dioxide $\left(\mathrm{NO}_{2}\right)$ emissions as of 2002 (Yu et al. 2009).

One of the key bottlenecks that cause traffic congestion is signalized intersections. Particularly, urban signalized intersections have been identified as a major source of vehicle emissions, as congestion along with complex vehicle activities including stop-and-go, deceleration, idling, and acceleration lead to more pollutant emissions (Pandian et al. 2009; Mahmod et al. 2013). Solutions are needed which can reduce intersection congestion and the resulting traffic-induced emission under
Corresponding author: Zhixia Li
E-mail: richard.li@louisville.edu
${ }^{*}$ Research was completed at TOPS Laboratory (University of Wisconsin-Madison).
${ }^{*}$ Guest Editor of the Special Issue of TRANSPORT on Smart and Sustainable Transport -
the manuscript was handled by one of Associate Editors, who made all decisions related to
the manuscript (including the choice of referees and the ultimate decision on the revision
and publishing).
Copyright $\odot 2015$ Vilnius Gediminas Technical University (VGTU) Press http://www.tandfonline.com/TRAN
Taylor \& Francis

Taylor \& Francis Group 
the rapidly increasing traffic demand in the future. Key to such solutions is keeping traffic moving through the intersections with reduced stops.

The availability of the maturing technologies of autonomous vehicles and Vehicle-To-Infrastructure (V2I) communications provides technical feasibility to develop solutions to improve mobility and sustainability of urban intersections. Particularly, autonomous vehicles with V2I capabilities make it possible to eliminate traditional traffic signals from the intersection, and can possibly increase intersection mobility substantially by fully utilizing the intersection time-space and reduce vehicle stops at intersections. Vehicle emissions and energy consumption are therefore potentially reduced due to the reduced number of vehicle stops at intersections. Additionally, from a safety perspective, considering that $90 \%$ of road crashes are attributed to driver errors (NHTSA 2013), autonomous vehicles are also potentially effective in reducing intersection related crashes.

In summary, a synergistic combination of autonomous vehicles and V2I communication technologies in intersection traffic control is promising in reducing vehicle stops, delays, energy consumption, vehicle emissions, and crashes at intersections, which may lead to solutions to sustainable next-generation intersections. This paper is aimed at quantifying the sustainability benefit offered by next-generation intersections that incorporate autonomous vehicles and V2I communication technologies. Specifically, the potential reduction in pollutant emission and energy consumption is evaluated and compared with traditional intersection control strategies.

\section{Literature Review}

Autonomous vehicles are vehicles without human intervention (in-vehicle or remote) and are capable of driving in highway systems by performing complex tasks such as merging, weaving, and driving through intersections. Many automotive manufacturers including General Motors, Ford, Mercedes-Benz, Volkswagen, Audi, BMW, Volvo and Cadillac have already begun testing their autonomous vehicle on highway systems (Wikipedia 2014a). Google is also developing and testing its Google driverless car (Wikipedia 2014b). As of 2012, Florida, Hawaii, Nevada, Oklahoma, and California of the US have legalized or are considering legalization of autonomous cars. All these facts indicate that the autonomous vehicles are set to appear on road in near future. Meanwhile, communication technologies have also achieved rapid advancements in recent years. In the US, Dedicated Short Range Communications (DSRC) protocol, which is widely applied in connected vehicle research, has been validated in terms of its capability in V2I and Vehicle-to-Vehicle (V2V) communications (Hu, Gharavi 2011; DoT 2014).

By employing either V2I or V2V communications, previous studies have investigated both centralized and decentralized strategies for managing autonomous vehicles at intersections (Alonso et al. 2011; Au et al. 2011; Ball, Dulay 2010; Dresner, Stone 2008a, 2008b; Fajardo et al. 2011; Quinlan et al. 2010; Shahidi et al. 2011; VanMiddlesworth et al. 2008; Vasirani, Ossowski 2009; Wu et al. 2007, 2010; Yan et al. 2008, 2009). Centralized control features an intersection controller that regulates the entire intersection. Vehicles only communicate with the central controller to get passing/stopping instructions. Decentralized control typically features negotiationbased control strategy, under which approaching vehicles from different directions communicate with each other to negotiate the passing sequence.

An evaluation study indicated that among all possible solutions to autonomous intersection control, the reservation-based centralized control had the best operational performance in terms of maximizing the intersection capacity and reducing number of vehicle stops and the delay (Wu et al. 2010). In reservation-based system, intersection is divided into a grid of $n$-by- $n$ tiles. An intersection central controller, which communicates with all approaching vehicles, decides the passing sequence based on whether certain intersection timespace (in terms of tiles at a certain time) that is needed for a vehicle to traverse through is already reserved by another vehicle (Dresner, Stone 2008a, 2008b; Fajardo et al. 2011; Quinlan et al. 2010; Shahidi et al. 2011; VanMiddlesworth et al. 2008).

In summary, the reservation-based centralized control has been proven to be more effective in reducing vehicle stops when compared with other intersection control strategies for autonomous vehicles. With reduced vehicle stops, the reservation-based control has great potential to reduce vehicle pollutant emission and energy consumption because energy- and emissionintensive behaviour such as stop-and-go, deceleration, idling and acceleration will less likely happen under reservation-based control. According to the review of literature, there is lack of research effort in developing and evaluating autonomous intersection control strategies from the perspective of enhancing sustainability specifically reduced emissions and energy consumption.

\section{Research Objectives}

This paper is intended to quantify the sustainability benefit from applying a novel control strategy that manages autonomous vehicles at intersection level. The control strategy applied in this research is an enhanced reservation-based intersection control algorithm (Autonomous Control of Urban TrAffic - ACUTA) for autonomous vehicles (Li et al. 2013b). The major research objective is two-fold:

- evaluate the sustainability effect from ACUTA measured in energy consumption and vehicle emissions;

- compare the single-tile ACUTA with 4-way stop control and multi-tile ACUTA with optimized signal control (pre-timed with minimal intersection delay) to quantify the sustainability enhancement in terms of reduction in energy consumption and vehicle emissions. 


\section{Sustainability-Enhancing Autonomous Intersection Control}

ACUTA is an enhanced autonomous intersection control algorithm developed based on the concept of FirstCome-First-Serve (FCFS) reservation-based protocol (Dresner, Stone 2008a, 2008b; Li et al. 2013a, 2013c). ACUTA introduced several operational enhancement strategies to further reduce vehicle stops and improve the mobility of the original reservation-based control protocol.

\subsection{The ACUTA Algorithm}

ACUTA utilizes a centralized control strategy that manages fully-autonomous vehicles at an intersection. All vehicles in ACUTA are autonomous and communicate only to an intersection controller. An intersection controller regulates the intersection by determining the passing sequence of all approaching vehicles. Specifically, intersection is divided into a mesh of $n$-by- $n$ tiles, as shown in Fig. 1, where $n$ is termed as granularity, which is tile density of the intersection mesh.

In ACUTA, each approaching vehicle automatically connects to the intersection controller via V2I communications after the vehicle enters the intersection controller's communication range, i.e., $600 \mathrm{ft}$ (183 m), which reflects a reasonable communication range based on the DSRC communication protocol (DoT 2014). When connected, the vehicle immediately starts to send the intersection controller an intersection reservation request along with the vehicle's location, speed and routing information (i.e., making a left/right turn or going straight), indicating the vehicle's intention to traverse the intersection.

The intersection controller processes the reservation request by running an internal simulation, in which the intersection controller computes the required timespaces for the requesting vehicle to traverse the intersection based on location, speed, maximum acceleration rate, and routing information provided by the requesting vehicle. In the internal simulation, intersection tiles that will be occupied by the requesting vehicle (e.g., the filled tiles as shown in Fig. 1) for every 0.1 second of the process the vehicle traversing the intersection. The internal simulation prefers a zero acceleration rate, as energy consumption is minimized if no acceleration is applied.

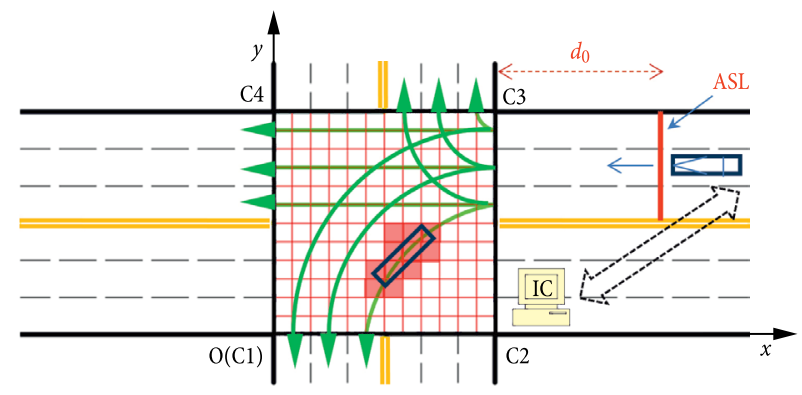

Fig. 1. Intersection mesh of tiles and example of vehicle's possible routing decisions (IC - Intersection Controller)
On the other hand, if acceleration is not considered, the vehicle may have smaller chance to obtain the reservation. Therefore, a balanced strategy is used in ACUTA as a solution to the aforementioned trade-off. The internal simulation first tries the zero acceleration. If conflicts exist between the resulting occupied intersection tiles with an existing reservation, alternative acceleration rates are considered in the internal simulation. If all alternative acceleration rates are tried out and conflicts still exist, the reservation request will be rejected; otherwise, the reservation request will be approved. The intersection controller automatically rejects requests from a vehicle that is following a vehicle that is without a reservation.

After making a decision to reject the reservation request, the intersection controller sends a rejection message to the requesting vehicle with a designated deceleration rate. A vehicle with a rejected reservation request will apply the designated deceleration rate and start to decelerate as soon as the rejection message is received. The vehicle keeps sending reservation requests until the request is finally approved by the intersection controller. In case the reservation request is approved, the intersection controller sends an approval message to the requesting vehicle along with a designated acceleration rate (can be zero) that will result in no conflicts with any existing reservations. Timestamps indicating when to end the acceleration and when to completely clear the intersection are also sent to the vehicle in the approval message. The approved vehicle will follow the acceleration instruction as soon as it receives the approval message until the vehicle completely clears the intersection.

A key process in the ACUTA algorithm was to determine each vehicle's central point location, vehicle boundary locations, and eventually which tiles are occupied by the vehicle. As shown in Fig. 2, a vehicle's path within the intersection was modelled as arcs whose centre coordinates were known.

Fig. 2a, 2b illustrate cases of left-turn and right-turn movements, respectively, with the arc centres denoted as $P$. For left-turn cases, assuming that the left-turn vehicle's central point reaches the boundary point $S\left(x_{s}, y_{s}\right)$ at time 0 , the coordinates of the vehicle's central point can be calculated using the following equation:

$$
\left\{\begin{array}{l}
x_{t}=x_{p}-R \cdot \sin (\alpha+\beta) \\
y_{t}=y_{p}+R \cdot \cos (\alpha+\beta)
\end{array}\right.
$$

where: $x_{t}-x$ coordinate of the vehicle's central point at time $t[\mathrm{~m}] ; y_{t}-y$ coordinate of the vehicle's central point at time $t[\mathrm{~m}] ; x_{p}-x$ coordinate of the turning arc's centre $[\mathrm{m}] ; y_{p}-y$ coordinate of the turning arc's centre $[\mathrm{m}] ; R=\sqrt{\left(x_{p}-x_{s}\right)^{2}+\left(y_{p}-y_{x}\right)^{2}}$ - the radius of the turning arc $[\mathrm{m}] ; \alpha=\frac{A}{R}[\operatorname{radian}] ; \beta=\arctan \left(\frac{\left|x_{p}-x_{s}\right|}{\left|y_{p}-y_{s}\right|}\right)$ [radian]; $x_{s}-x$ coordinate of the vehicle's central point at time $0[\mathrm{~m}] ; y_{s}-y$ coordinate of the vehicle's central point at time $0[\mathrm{~m}] ; A=v \cdot t$ - the arc length $[\mathrm{m}] ; v-$ speed of the vehicle when it is in the intersection $[\mathrm{m} / \mathrm{s}]$; 
a)

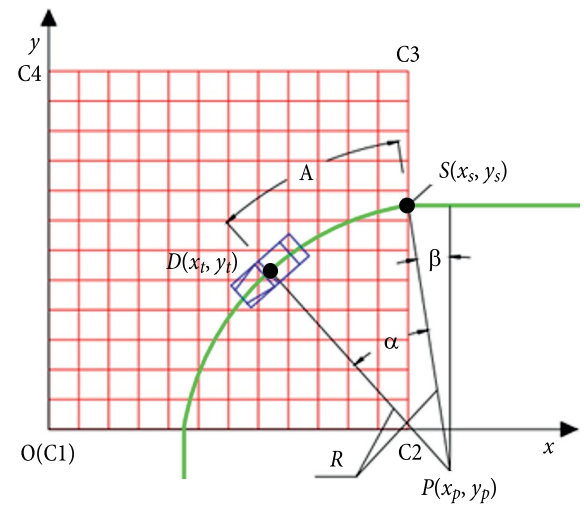

c)

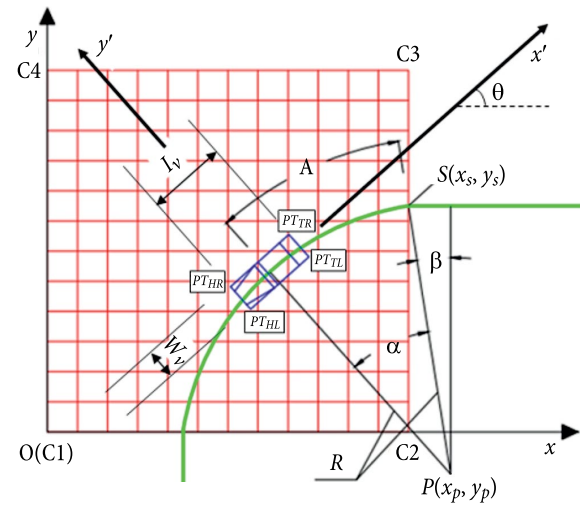

b)

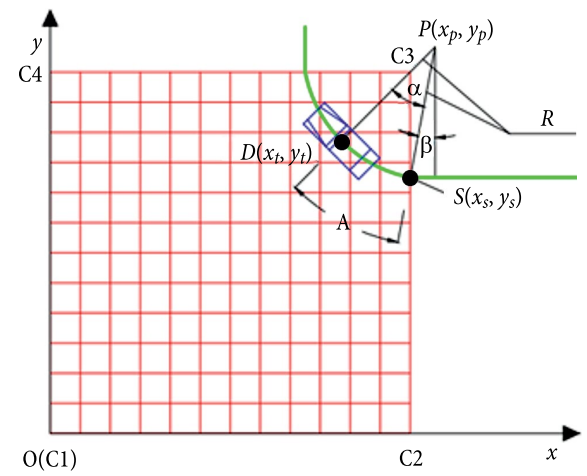

d)

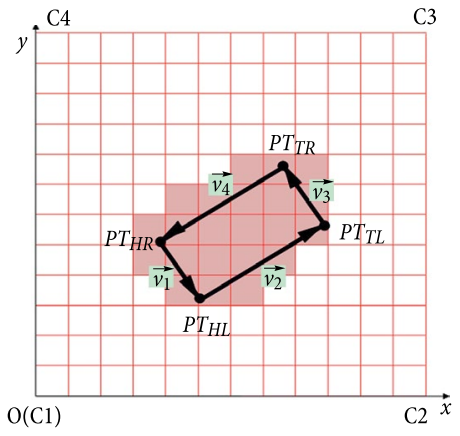

Fig. 2. Algorithms of determining the vehicle boundary and tiles occupied

$t$ - any time when the vehicle's central point is within the intersection $[s]$.

Similarly, assuming that the right-turn vehicle's central point reaches the boundary point $S\left(x_{s}, y_{s}\right)$ at time 0 , the coordinates of the vehicle's central point can be calculated using the following equation:

$$
\left\{\begin{array}{l}
x_{t}=x_{p}-R \cdot \sin (\alpha+\beta) ; \\
y_{t}=y_{p}-R \cdot \cos (\alpha+\beta),
\end{array}\right.
$$

where: $x_{t}-x$ coordinate of the vehicle's central point at time $t[\mathrm{~m}] ; y_{t}-y$ coordinate of the vehicle's central point at time $t[\mathrm{~m}] ; x_{p}-x$ coordinate of the turning arc's centre $[\mathrm{m}] ; y_{p}-y$ coordinate of the turning arc's centre $[\mathrm{m}]$; $R=\sqrt{\left(x_{p}-x_{s}\right)^{2}+\left(y_{p}-y_{x}\right)^{2}}$ - the radius of the turning $\operatorname{arc}[\mathrm{m}] ; \alpha=\frac{A}{R}$ [radian]; $\beta=\arctan \left(\frac{\left|x_{p}-x_{s}\right|}{\left|y_{p}-y_{s}\right|}\right)$ [radian]; $x_{s}-x$ coordinate of the vehicle's central point at time $0[\mathrm{~m}] ; y_{s}-y$ coordinate of the vehicle's central point at time $0[\mathrm{~m}] ; A=v \cdot t$ - the arc length $[\mathrm{m}] ; v$ - speed of the vehicle when it is in the intersection $[\mathrm{m} / \mathrm{s}] ; t-$ any time when the vehicle's central point is within the intersection $[\mathrm{s}]$.

Once the central point location was determined, the locations of the vehicle boundaries were determined. Fig. $2 c$ illustrates the locations vehicle's vertices. The length of the rectangle is $l_{v}$ and the width of the rectangle is $w_{v}$, equalling to the corresponding vehicle's length and width, respectively. The vertices of the rectangle rep- resent the four corners of the vehicle: head left $P T_{H L}$, head right $P T_{H R}$, tail left $P T_{T L}$ and tail right $P T_{T R}$. Given a central point coordinate $\left(x^{\prime}, y^{\prime}\right)$ in local coordinate system, its coordinates in the global system $(x, y)$ can be calculated using a coordinate rotation followed by a coordinate transfer. The formula is given below:

$$
\left[\begin{array}{l}
x \\
y
\end{array}\right]=\left[\begin{array}{cc}
\cos \theta & -\sin \theta \\
\sin \theta & \cos \theta
\end{array}\right] \cdot\left[\begin{array}{l}
x^{\prime} \\
y^{\prime}
\end{array}\right]+\left[\begin{array}{l}
x_{t} \\
y_{t}
\end{array}\right],(3)
$$

where: $x_{t}-x$ coordinate of the vehicle's central point at time $t[\mathrm{~m}] ; y_{t}-y$ coordinate of the vehicle's central point at time $t[\mathrm{~m}] ; \theta$ - the smallest angle measured counterclockwise from the $x$ axis to the $x^{\prime}$ axis. In the case of Fig. 2c, $\theta=\alpha+\beta$ [radian].

Based on Eq. (3), the global coordinates of the vehicle vertices can be easily converted from their local coordinates. For example, the local coordinates of the $P T_{H R}$ vertex are $\left(x^{\prime}=-\frac{l_{v}}{2}, y^{\prime}=\frac{w_{v}}{2}\right)$. By substituting $x^{\prime}$ and $y^{\prime}$ with $-\frac{l_{v}}{2}$ and $\frac{w_{v}}{2}$ in Eq. (3), the global coordinates of $P T_{H R}$ are $\left(x=-\frac{l_{v} \cdot \cos \theta+w_{v} \cdot \sin \theta}{2}+x_{t}\right.$, $\left.y=-\frac{l_{v} \cdot \sin \theta-w_{v} \cdot \cos \theta}{2}+y_{t}\right)$.

When the coordinates of a vehicle's vertices are known, which tiles are occupied by the vehicle were then determined. Fig. 2d depicts a vehicle with all occupied tiles highlighted in red. The criterion to de- 
termine whether a tile is occupied by a vehicle is: at least one vertex of the tile is inside the vehicle rectangle. In ACUTA, a vector based method is used to decide whether a point falls in the vehicle rectangle. As shown in Fig. 2d, four vectors are defined counter-clockwise along the vehicle rectangle. The four vectors are $\overrightarrow{v_{1}}\left(P T_{H R} \rightarrow P T_{H L}\right), \quad \overrightarrow{v_{2}}\left(P T_{H L} \rightarrow P T_{T L}\right)$, $\overrightarrow{v_{3}}\left(P T_{T L} \rightarrow P T_{T R}\right)$ and $\overrightarrow{v_{4}}\left(P T_{T R} \rightarrow P T_{H R}\right)$. A point is within the vehicle rectangle only if it falls to the left of all four vectors. Given a point $p\left(x_{0}, y_{0}\right)$ and a vector $\overrightarrow{v_{i}}\left(\left(x_{\text {start }}, y_{\text {start }}\right) \rightarrow\left(x_{\text {end }}, y_{\text {end }}\right)\right), p$ falls to the left of $\overrightarrow{v_{i}}$ only when the following equation is satisfied:

$$
\left(x_{0}-x_{\text {start }}\right) \cdot\left(y_{\text {end }}-y_{0}\right)-\left(x_{\text {end }}-x_{0}\right) \cdot\left(y_{0}-y_{\text {start }}\right)<0 \text {, }
$$

where: $x_{0}-x$ coordinate of the testing point $[\mathrm{m}] ; y_{0}-y$ coordinate of the testing point $[\mathrm{m}] ; x_{\text {start }}-x$ coordinate of the vector's start point $[\mathrm{m}] ; y_{\text {start }}-y$ coordinate of the vector's start point $[\mathrm{m}] ; x_{\text {end }}-x$ coordinate of the vector's end point $[\mathrm{m}] ; y_{\text {end }}-y$ coordinate of the vector's end point $[\mathrm{m}]$.

As a tile is bounded by two horizontal lines and two vertical lines, the following equation was used to determine whether a vertex of the vehicle rectangle falls in a tile:

$$
\left\{\begin{array}{l}
x_{\text {low }}<x_{0}<x_{\text {high }} \\
y_{\text {low }}<y_{0}<y_{\text {high }}
\end{array},\right.
$$

where: $x_{0}-x$ coordinate of the testing point $[\mathrm{m}] ; y_{0}-y$ coordinate of the testing point $[\mathrm{m}] ; x_{\text {low }}-$ shared $x$ coordinate of left vertices of the tile $[\mathrm{m}] ; y_{\text {low }}$ - shared $y$ coordinate of bottom vertices of the tile [m]; $x_{\text {high }}-\operatorname{shared} x$ coordinate of right vertices of the tile $[\mathrm{m}] ; y_{\text {high }}-$ shared $y$ coordinate of top vertices of the tile [m].

In summary, given a tile and a vehicle rectangle, Eqs (4) or (5) were used to determine whether the vehicle rectangle has occupied the tile. If any of the four vertices of the tile satisfies Eqs (4) or (5), the tile is considered occupied by the vehicle.

\subsection{Enhancement ACUTA Strategies to Minimize Vehicle Stops and Improve Sustainability}

In addition to sustainability-friendly strategies mentioned in Section 3.1, that reduce occurrences of vehicle accelerations, such as (1) the zero initial acceleration rate in the internal simulation; and (2) turning from any lanes to avoid lane changes, ACUTA introduced the following two additional enhancement strategies to further reduce vehicle stops at intersection and in turn benefits the reduction of vehicle emission and energy consumption.

- Non-Deceleration Zone (NDZ);

- Priority Reservation (PR).

Non-Deceleration Zone (NDZ): NDZ defines an open upstream-end zone from an advance location from the intersection. There is no upstream boundary for NDZ. Vehicles in NDZ do not need to decelerate even their reservation requests are rejected. The downstream boundary of NDZ is at a location that can ensure that a vehicle can stop with a reasonably high deceleration rate (e.g. $15 \mathrm{ft} / \mathrm{s}^{2}$ ). The downstream boundary of NDZ is a configurable parameter, which can be set as a specific location, which can assure a comfortable deceleration rate. NDZ can help a vehicle continue to maintain a high traveling speed even though its reservation request is rejected. This gives the vehicle a better chance of obtaining a reservation with a later request and meanwhile reduces possibility of vehicle stops.

Priority Reservation (PR) for queuing vehicles: the $P R$ gives queuing vehicles a better chance to get their reservation requests approved by prioritizing processing of their reservation requests by the intersection manager. Queueing vehicles are defined as vehicles traveling at a speed less than about $10 \mathrm{~km} / \mathrm{hr}$. Once PR is activated, vehicles in slowly moving queue have priority for placing reservation requests, which reduces the possibility of vehicle stops at intersections, hence potentially improves vehicle emission and energy consumption.

\subsection{Type of ACUTA Operations}

Granularity of the intersection mesh is one of the most important parameters in the ACUTA system. If the granularity is set to one, the entire intersection is undivided and only one vehicle can occupy the entire intersection at one time. The system in this case is termed as SingleTile ACUTA system. When the granularity is greater than one, the system is termed as Multi-Tile ACUTA system.

\subsection{Modelling ACUTA in VISSIM}

VISSIM is one the most widely used standard microscopic simulation platforms that are used in modelling intersections. Particularly, VISSIM offers flexible customization functions to facilitate building different special applications through VISSIM External Driver Model and COM extensions. All these functions facilitated implement V2I communications and the ACUTA algorithm. The External Driver Model bypasses and replaces VISSIM's internal driving behaviours. During a simulation run, VISSIM calls the External Driver Model DLL at every simulation step to pass the current state of each vehicle to the DLL. The intersection controller was built in the External Driver Model DLL, which collects each vehicle's speed, location, vehicle class, maximum acceleration rate, length, width, and many other parameters pertaining to the particular vehicle at each simulation step. The intersection controller processes all reservation requests at the beginning of each simulation step ( $0.1 \mathrm{~second} / \mathrm{step})$, and passes its decision and the suggested acceleration/deceleration rate to the drivers in the same simulation step. The vehicles then pass their acceleration/deceleration rate back to VISSIM at the same simulation step, thus the real-time control of each vehicle's acceleration rate is realized.

The simulated intersection where the ACUTA operates at is a four-legged intersection with three lanes per direction, as shown in Fig. 3. Vehicles can turn from any lane in the ACUTA intersection. Therefore, no enroute lane change is required for turning vehicles, thus 


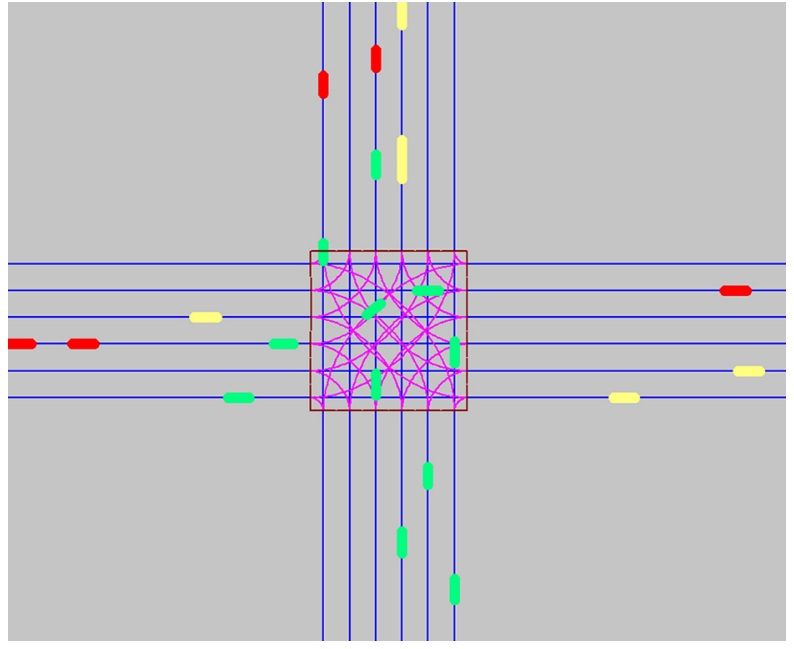

Fig. 3. Modelled ACUTA in VISSIM

minimizes the delays due to the conflicts caused by vehicle lane change manoeuvres. Because of the absence of lane changes, each lane in the simulation model is built as a separate link to simplify the complexity of the simulation model. Roadways are assumed to have zero grades in the simulation model.

Each approach of the intersection is more than $2000 \mathrm{ft}(610 \mathrm{~m})$ long with a fixed lane width of $12 \mathrm{ft}$ $(3.7 \mathrm{~m})$. The volume input of each lane is identical, trying to create balanced traffic demands from all lanes of the intersection. Each lane has three routing decisions: left turn, straight, and right turn. The volume assignments to the routing decisions are the same for all lanes, namely $25 \%$ for left turn, $60 \%$ for through, and $15 \%$ for right turn. The vehicle composition takes $93 \%$ passenger cars and 7\% heavy vehicles. The speed distribution of traffic is also fixed at a setting equivalent to the $30 \mathrm{mph}$ (48 kph) speed limit. No priority rules, conflict areas, desired speed decisions, reduced speed areas, traffic signals, or stop signs are used in the simulation model, because the traffic control of the entire intersection is governed by the intersection controller only. Vehicle maximum deceleration rate follows the default maximum deceleration rate distribution predefined in VISSIM. Vehicle maximum acceleration rate is set as $9.8 \mathrm{ft} / \mathrm{s}^{2}\left(3.0 \mathrm{~m} / \mathrm{s}^{2}\right)$.

In Fig. 3, red vehicles are vehicles that do not have a reservation; green vehicles are vehicles that have a reservation and are in the process of passing the intersection; and yellow vehicles are those that have already cleared the intersection.

\section{Estimating Emission and Energy Consumption of the ACUTA Intersection}

\subsection{Method to Estimate Emission and Energy Consumption}

MOVES is US Environmental Protection Agency's (EPA) state-of-the-art tool for estimating emissions and energy consumption from highway vehicles, with its first version released in 2004 (EPA 2010). Different from the EPA's older model, such as MOBILE, MOVES uses a modal emission approach, which classifies vehicles at different time stamps into different operating modes. MOVES emission simulation can be achieved at national level (macroscopic), county level and project level (microscopic). For a project level analysis, MOVES provides three methods for inputting traffic activity (EPA 2010), including:

- link average speed;

- link drive schedule;

- operating mode distribution.

Among the three methods under MOVES project level, the method of operating mode distribution requires the most detailed input data, which in turn leads to the most accurate emission estimation (EPA 2010). For evaluating the ACUTA intersection, the operating mode distribution method was used. As MOVES requires input of the distribution of operating mode rather than trajectories, several computational and data manipulation steps were needed. Specifically, the following three spreadsheets are needed as input by MOVES:

- link table that contains link number, length, volume, grade and average speed;

- link source type table that contains the proportion of different types of vehicles in the link;

- operating mode distribution table that contains the proportion of each type of operating mode bins for each link within a defined study period.

To ultimately generate these spreadsheets, interim variables including acceleration rate, Vehicle Specific Power (VSP), and operating mode, need to be computed. The following sections discuss how these interim variables were computed for the ACUTA intersection.

\section{Computation of VSP}

VSP is a significant factor for determining a vehicle's operating mode. The equation of VSP is a mathematical representation of engine load against aerodynamic drag, acceleration, rolling resistance, plus the kinetic and potential energies of the vehicle, all divided by the mass of the vehicle (Jiménez-Palacios 1999). Its original equation is in the following form:

$$
V S P=v \cdot\left(1.1 \cdot a+g \cdot \sin \theta+g \cdot C_{r}\right)+\frac{\rho_{a} \cdot C_{d} \cdot A_{f} \cdot v^{3}}{2 \cdot M},
$$

where: $v$ - vehicle speed $[\mathrm{m} / \mathrm{s}] ; a$ - vehicle acceleration rate $\left[\mathrm{m} / \mathrm{s}^{2}\right] ; g-$ acceleration due to gravity $\left[9.81 \mathrm{~m} / \mathrm{s}^{2}\right]$; $\theta$ - grade; $C_{r}$ - coefficient of rolling resistance; $\rho_{a}$ - density of air $\left[\mathrm{kg} / \mathrm{m}^{3}\right] ; C_{d}$ - coefficient of aerodynamic drag; $A_{f}$ - frontal area of vehicle $\left[\mathrm{m}^{2}\right] ; M-$ mass of vehicle $[\mathrm{kg}]$.

In practice, a generic set of coefficients values for estimating VSP for a typical light duty fleet is applied as a useful basis for characterization (Frey et al. 2006; Yao et al. 2013). VSP is calculated based on only measured speed, acceleration and road grade. The VSP values for light duty vehicles are calculated using the following equation (Frey et al. 2006; Yao et al. 2013):

$$
V S P=v \cdot(1.1 \cdot a+9.81 \cdot \text { grade }+0.132)+0.000302 \cdot v^{3},
$$

where: $v$ - vehicle speed $[\mathrm{m} / \mathrm{s}] ; a$ - vehicle acceleration rate $\left[\mathrm{m} / \mathrm{s}^{2}\right]$; grade - vehicle vertical rise divided by the horizontal run [\%]. 


\section{Computation of Operating Mode}

MOVES is based on operating mode distribution to determine emission rates for a project level analysis. In total, MOVES has defined 23 operating modes for estimation of vehicle running exhaust. A vehicle's operating mode is determined by the vehicle's speed and VSP. The detailed definition of each operating mode bins are defined in MOVES User Manual (EPA 2010).

\section{Estimation of Emission and Energy Consumption}

With operating mode distribution table, the link table, and the link source type table, MOVES can estimate emission and energy consumption on all the links defined in the Link table. Note that MOVES also requires other data, such as meteorological data, vehicle age, and fuel type. Default values were used for these data elements. Although MOVES is able to simulate emission by considering several factors, including running exhaust, start exhaust, and tire wear, only running exhaust was considered in this method as it is the most significant type of emission at intersections. In this project, the following elements were estimated using MOVES.

- carbon monoxide;

- PM 2.5;

- energy consumption.

\subsection{Estimating ACUTA Sustainability Performance from the Simulation Model}

The ACUTA intersection modelled in VISSIM can output trajectory data for each vehicle in the simulation at every 0.1 simulation second. The trajectory data include vehicle speed and vehicle acceleration rate. Both are data elements needed to compute VSP per Eq. (7). Links of the ACUTA intersection simulation model were divided in order to define the MOVES links. Fig. 4 illustrates how MOVES links were defined for the ACUTA intersection. Table 1 lists all links' definition and distance range.

The total amount of vehicle emission/energy consumption from all links of the ACUTA intersection was simply computed using the following equation:

$$
E=\sum_{i=1}^{n} E_{i}
$$

where: $E$ - total amount of emission/energy for all links in an hour; $N$ - number of links; $E_{i}$ - amount of emission/energy for link $i$ in an hour.

\section{Results of Sustainability Performance of ACUTA}

Sustainability evaluation of the ACUTA was performed by converting the trajectory output from VISSIM into MOVES inputs, and running MOVES to obtain the emission and energy consumption outputs. Both the Multi-tile ACUTA and single-tile ACUTA were evaluated. To investigate the sustainability benefit from implementing ACUTA, sustainability performance of traditional traffic control such as signalized control and four-way stop control were compared with ACUTA.

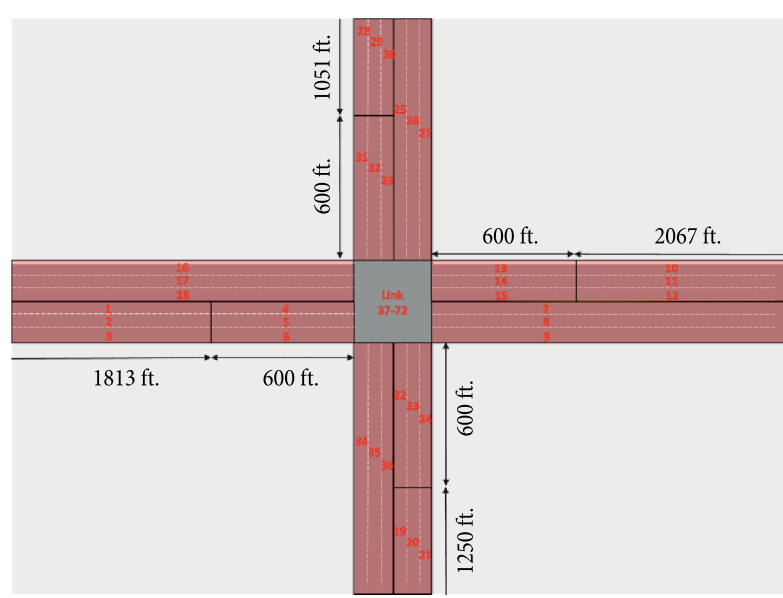

Fig. 4. MOVES link definition for the ACUTA intersection

Table 1. MOVEs Link definitions and locations.

\begin{tabular}{ccl}
\hline $\begin{array}{c}\text { MOVES } \\
\text { link } \\
\text { ID }\end{array}$ & $\begin{array}{c}\text { Link } \\
\text { length } \\
{[\mathrm{ft}]}\end{array}$ & \multicolumn{1}{l}{ Link description } \\
\hline $1-3$ & 1813 & EB cruise links for EB lanes 1-3 \\
\hline $4-6$ & 600 & EB deceleration links for EB lanes 1-3 \\
\hline $7-9$ & 2667 & EB acceleration links for EB lanes 1-3 \\
\hline $10-12$ & 2067 & WB cruise Links for WB lanes 1-3 \\
\hline $13-15$ & 600 & WB deceleration Links for WB lanes 1-3 \\
\hline $16-18$ & 2413 & WB acceleration Links for WB lanes 1-3 \\
\hline $19-21$ & 1250 & NB cruise Links for EB lanes 1-3 \\
\hline $22-24$ & 600 & NB deceleration Links for EB lanes 1-3 \\
\hline $25-27$ & 1651 & NB acceleration Links for EB lanes 1-3 \\
\hline $28-30$ & 1051 & SB cruise Links for EB lanes 1-3 \\
\hline $31-33$ & 600 & SB deceleration Links for EB lanes 1-3 \\
\hline $34-36$ & 1850 & SB acceleration Links for EB lanes 1-3 \\
\hline $37-72$ & Varies & All links inside intersection \\
\hline
\end{tabular}

Multi-tile ACUTA was compared with the optimized signalized control while the single-tile ACUTA was compared with the four-way stop control. To do this, the ACUTA intersection simulation model was converted into a signalized intersection model and a four-way stop intersection model. For the multi-tile ACUTA evaluated, the granularity used was 12 .

\subsection{Multi-Tile ACUTA vs. Optimized Signal Control}

The optimized signal control reflects pre-timed signal with signal timing being optimized to achieve the minimal intersection delay based on the volume inputs. Highway Capacity Software 2010 was used to obtain the optimized timing (HCS 2010; HCM 2010). The optimization was performed as adaptive signal control strategies that automatically change signal timing based on traffic demand via loop detections typically perform much better than the pre-timed signal control. The traffic signal was optimized to achieve a similar (theoretically may be better) performance that can be achieved by making the 
signal control adaptive, as the optimization was based on the specific input traffic demands and the objective of the optimization was obtaining the minimal intersection delay. Therefore, ACUTA was compared to the best performance that is achievable by an isolated intersection.

Three traffic volume conditions, 300, 900, 1800 veh/hr/approach, which represent low, moderate, and high, respectively, were used to evaluate the multi-tile ACUTA's and the optimized signal control's sustainability performance. All approaches of the intersection use the same volume input. For each traffic demand condition, five simulation runs with different random seeds were performed for ACUTA and for signal control. Each simulation run lasted 2,100 seconds, with the first 300 warm-up seconds dropped from the evaluation.

Table 2 summarizes the detailed optimized signal timing based on the input traffic demands. Tables 3-5 summarize the comparison between multi-tile ACUTA and signalized control in terms of the total amounts of CO emission, PM 2.5 emission, and energy consumption for the entire intersection in an hour.

The comparisons show that replacing signal control with multi-tile ACUTA reduces CO emission by $8.6,4.9$ and $3.0 \%$ and PM 2.5 emission by $6.0,5.2$ and $2.6 \%$ under low, moderate and high volume conditions, respectively. At the same time, the energy consumption was also reduced by $8.6,4.9$ and $3.0 \%$ under low, moderate and high volume conditions.

\subsection{Single-Tile ACUTA vs. Optimized Four-Way Stop Control}

A single-tile ACUTA intersection has an undivided intersection mesh, which means only one vehicle can occupy the entire intersection at a specific instant. Therefore, the single-tile ACUTA system is relatively easier to implement than the multi-tile ACUTA system. The single-tile ACUTA is hence a promising replacement for the Four-Way Stop Control (4WSC), considering that the operational characteristics of both the singletile ACUTA and the four-way stop control are analogous. The major difference between these two control strategies is that the vehicles in the ACUTA system do not necessarily need to stop before their entry into the intersection. However, at a four-way stop intersection, whoever stops at the stop line first gets the right of way.

Table 2. Optimized signal timings for traffic demand evaluated

\begin{tabular}{|c|c|c|c|c|c|c|c|c|}
\hline \multirow{3}{*}{$\begin{array}{l}\text { Approach traffic demand } \\
\text { [veh/hr/approach] }\end{array}$} & \multicolumn{3}{|c|}{ Approach demand by movement [veh/hr] } & \multicolumn{5}{|c|}{ Signal timing plan } \\
\hline & \multirow[b]{2}{*}{ LT } & \multirow[b]{2}{*}{ Thru } & \multirow[b]{2}{*}{ RT } & \multirow[b]{2}{*}{ Cycle length $[s]$} & \multicolumn{4}{|c|}{ Phase timing [s] } \\
\hline & & & & & - & 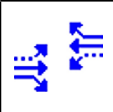 & 5 & 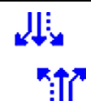 \\
\hline High volume: 300 & 75 & 180 & 45 & 40 & 6 & 6 & 6 & 6 \\
\hline Moderate volume: 900 & 225 & 540 & 135 & 60 & 6 & 16 & 6 & 16 \\
\hline High volume: 1800 & 450 & 1080 & 270 & 110 & 12 & 35 & 12 & 35 \\
\hline
\end{tabular}

Table 3. Comparison of CO emission between multi-tile ACUTA and signal control

\begin{tabular}{l|rc|c}
\hline \multirow{2}{*}{$\begin{array}{c}\text { Approach traffic demand } \\
\text { [veh/hr/approach] }\end{array}$} & \multicolumn{2}{|c|}{ CO $[\mathrm{g}]$} & \multirow{2}{*}{ Improvement by implementing ACUTA } \\
\cline { 2 - 3 } & Signal & ACUTA & $5.6 \%$ \\
\hline Low volume: 300 & 12159 & 11482 & $4.9 \%$ \\
\hline Moderate volume: 900 & 49906 & 47453 & $3.0 \%$ \\
\hline high volume: 1800 & 73860 & 71678 & \\
\hline
\end{tabular}

Table 4. Comparison of PM 2.5 emission between multi-tile ACUTA and signal control

\begin{tabular}{l|cc|c}
\hline \multirow{2}{*}{$\begin{array}{c}\text { Approach traffic demand } \\
\text { [veh/hr/approach] }\end{array}$} & \multicolumn{2}{c|}{ PM 2.5 [g] } & \multirow{2}{*}{ Improvement by implementing ACUTA } \\
\cline { 2 - 3 } & Signal & ACUTA & $5.8 \%$ \\
\hline Low volume: 300 & 29.2 & 27.5 & $5.2 \%$ \\
\hline Moderate volume: 900 & 129.3 & 122.5 & $2.6 \%$ \\
\hline High volume: 1800 & 176.6 & 172.1 & \\
\hline
\end{tabular}

Table 5. Comparison of energy consumption between multi-tile ACUTA and signal control

\begin{tabular}{l|cc|c}
\hline \multirow{2}{*}{$\begin{array}{c}\text { Approach traffic demand } \\
\text { [veh/hr/approach] }\end{array}$} & \multicolumn{2}{|c|}{ Energy Consumption [KJ] } & \multirow{2}{*}{ Improvement by implementing ACUTA } \\
\cline { 2 - 3 } & Signal & ACUTA & $3.8 \%$ \\
\hline Low volume: 300 & 9981245 & 9601578 & $4.9 \%$ \\
\hline Moderate volume: 900 & 29143648 & 27723932 & $11.9 \%$ \\
\hline High volume: 1800 & 59948005 & 52806697 & \\
\hline
\end{tabular}


Three traffic volume conditions, 75, 150, $225 \mathrm{veh} /$ $\mathrm{hr} /$ approach, which represent low, moderate, and high volumes, respectively, were used to evaluate the singletile ACUTA's and the four-way stop control's sustainability performance. All approaches of the intersection use the same volume input. For each traffic demand condition, five simulation runs with different random seeds were performed for ACUTA and for 4WSC. Each simulation run lasted 2,100 seconds, with the first 300 warm-up seconds dropped from the evaluation.

Tables 6 through 8 summarize the comparison results in terms of the total amounts of CO emission, PM 2.5 emission, and energy consumption for the entire intersection in an hour.

The comparisons indicate that converting four-way stop into single-tile ACUTA reduces $\mathrm{CO}$ emission by $14.3,15.2$ and $16.8 \%$ and PM 2.5 emission by $12.2,16.5$ and $18.3 \%$ under low, moderate and high volume conditions, respectively. Meanwhile, the energy consumption was also reduced by $16.2,15.7$ and $14.6 \%$ under low, moderate and high volume conditions.

\section{Discussions on Corridor Extension of ACUTA}

The sustainability performance of the ACUTA algorithm when implemented at an isolated intersection has been extensively investigated in the previous sections. In a long run, the sustainability benefit from autonomous vehicles and ACUTA can be enhanced if the ACUTA can be extended to a corridor and eventually to a network level. This section aims at exploring extending the ACUTA to a corridor level, where adjacent intersections are located with a certain distance.

The ACUTA algorithm is designed to manage traffic for an isolated intersection. The algorithm is generic across different intersections when the geometric information of the intersection is known. The deployment of the ACUTA at an isolated intersection only requires configuring the ACUTA with the specific geometric information pertaining to that particular intersection. Therefore, a simple approach for extending the ACUTA to the corridor level is to treat each intersection along the corridor as isolated intersections and directly deploy the isolated-intersection-based ACTUA at each intersection of that corridor. The concept of this approach is illustrated by Fig. 5 .

As shown in Fig. 5, each intersection along the corridor has a separate ACUTA intersection manager deployed. There is no communication or coordination between these adjacent intersection managers. Each intersection manager independently processes the reservation requests from the approachinsg vehicles that enter the communication range of its managed intersection. On the other hand, coordination of closely spaced ACUTA intersections could reduce stops because the approaching vehicles' speed and location information would be received by the downstream intersection man-

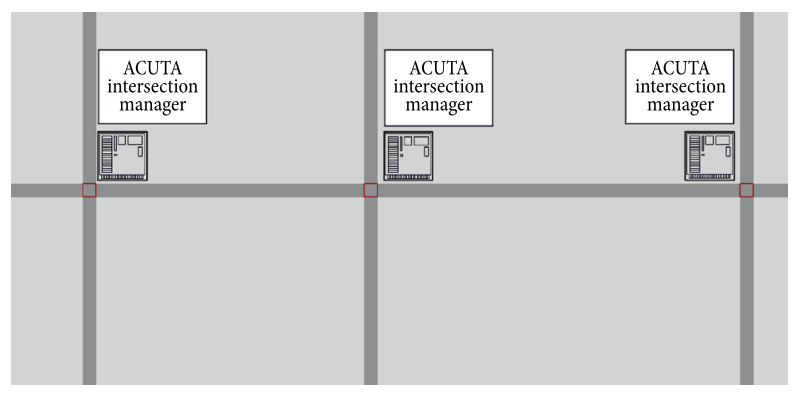

Fig. 5. Extension of the ACUTA to corridor level

Table 6. Comparison of CO emission between single-tile ACUTA and four-way stop control

\begin{tabular}{|c|c|c|c|}
\hline \multirow{2}{*}{$\begin{array}{l}\text { Approach traffic demand } \\
\text { [veh/hr/approach] }\end{array}$} & \multicolumn{2}{|c|}{$\mathrm{CO}[\mathrm{g}]$} & \multirow{2}{*}{ Improvement by implementing ACUTA } \\
\hline & 4WSC & ACUTA & \\
\hline Low volume: 75 & 4727 & 4053 & $14.3 \%$ \\
\hline Moderate volume: 150 & 9923 & 8416 & $15.2 \%$ \\
\hline High volume: 225 & 14846 & 12352 & $16.8 \%$ \\
\hline
\end{tabular}

Table 7. Comparison of PM 2.5 emission between single-tile ACUTA and four-way stop control.

\begin{tabular}{l|cc|c}
\hline \multirow{2}{*}{$\begin{array}{c}\text { Approach traffic demand } \\
\text { [veh/hr/approach] }\end{array}$} & \multicolumn{2}{|c|}{ PM 2.5 [g] } & Improvement by implementing ACUTA \\
\cline { 2 - 3 } & 4WSC & ACUTA & $12.2 \%$ \\
\hline Low volume: 75 & 26.4 & 22.0 & 16.7 \\
\hline Moderate volume: 150 & 39.2 & 32.0 & $18.3 \%$ \\
\hline High volume: 225 & & \\
\hline
\end{tabular}

Table 8. Comparison of energy consumption between single-tile ACUTA and four-way stop control

\begin{tabular}{l|cc|c}
\hline \multirow{2}{*}{$\begin{array}{c}\text { Approach traffic demand } \\
\text { [veh/hr/approach] }\end{array}$} & \multicolumn{2}{|c|}{ Energy Consumption [KJ] } & \multirow{2}{*}{ Improvement by implementing ACUTA } \\
\cline { 2 - 3 } & 4WSC & ACUTA & $16.2 \%$ \\
\hline Low volume: 75 & 2711023 & 2273029 & $15.7 \%$ \\
\hline Moderate volume: 150 & 5562711 & 4688058 & $14.6 \%$ \\
\hline High volume: 225 & 8011424 & 6839443 & \\
\hline
\end{tabular}


ager from the upstream intersection manager before the vehicles would enter the communication range of the downstream intersection. In this case, the reservation request would be pre-placed by a vehicle before the vehicle enters the communication range of the downstream intersection. Optimization strategies could also be applied to achieve minimized system emissions with the intercommunication mechanism between the intersections. The sustainability effects of corridor-based coordination and optimization will be investigated in future research.

\section{Conclusions}

The sustainability comparison between ACUTA and traditional intersection traffic control revealed that the autonomous vehicle and V2I communication technologies empowered ACUTA has great potential in reducing both pollutant emission and energy consumption at intersections. Specific benefits of implementing ACUTA are summarized as follows:

- Compared with optimized signal control, multitile ACUTA reduces CO and PM 2.5 emissions by about $5 \%$ under low to moderate volume conditions and by about $3 \%$ under high volume condition. Meanwhile, energy consumption is reduced by about $4 \%$ under low to moderate volume conditions and by about $12 \%$ under high volume condition.

- Compared with four-way stop control, singletile ACUTA reduces CO and PM 2.5 emissions as well as energy consumption all by about $15 \%$ under any prevailing volume conditions.

In conclusions, when applied appropriately, the next-generation vehicle technologies such as autonomous vehicle and V2I communications can bring great sustainability benefits at urban intersections, especially when both technologies are applied in combination. ACUTA is one particular example of such applications. The benefit in reducing emission and energy consumption majorly comes from the reduced number of vehicle stops at the ACUTA intersection. Future research will be focused on exploring avenues that can optimize the ACUTA algorithm by maximizing the sustainability benefit and evaluating the extended ACUTA to a corridor level.

\section{Acknowledgements}

This work was partially supported by US Department of Transportation (DoT) through the National Center for Freight and Infrastructure Research and Education (CFIRE).

\section{References}

Alonso, J.; Milanés, V.; Pérez, J.; Onieva, E.; González, C.; De Pedro, T. 2011. Autonomous vehicle control systems for safe crossroads, Transportation Research Part C: Emerging Technologies 19(6): 1095-1110.

http://dx.doi.org/10.1016/j.trc.2011.06.002
Au, T.-C.; Shahidi, N.; Stone, P. 2011. Enforcing liveness in autonomous traffic management, in Proceedings of the Twenty-Fifth AAAI Conference on Artificial Intelligence, 7-11 August 2011, San Francisco, CA, 2: 1317-1322. Available from Internet: http://www.aaai.org/Library/AAAI/aaai11 contents.php.

Ball, R.; Dulay, N. 2010. Enhancing traffic intersection control with intelligent objects, in First International Workshop on the Urban Internet of Things (Urban IOT 2010), 29 November - 1 December 2010, Tokyo, Japan.

CamSys. 2005. An Initial Assessment of Freight Bottlenecks on Highways. Prepared for Federal Highway Administration, Office of Transportation Policy Studies; prepared by Cambridge Systematics, Inc. (CamSys) in association with Battelle Memorial Institute, Columbus, Ohio. 125 p. Available from Internet: http://www.fhwa.dot.gov/policy/otps/bottlenecks/bottlenecks.pdf.

DoT. 2014. DSRC: The Future of Safer Driving. US Department of Transportation. Available at: http://www.its.dot.gov

Dresner, K.; Stone, P. 2008a. A multiagent approach to autonomous intersection management, Journal of Artificial Intelligence Research 31: 591-656.

http://dx.doi.org/10.1613/jair.2502

Dresner, K.; Stone, P. 2008b. Mitigating catastrophic failure at intersections of autonomous vehicles, in AAMAS'08: Proceedings of the 7th International Joint Conference on Autonomous Agents and Multiagent Systems, 12-16 May 2008, Estoril, Portugal, 3: 1393-1396.

EPA. 2010. Motor Vehicle Emission Simulator (MOVES) 2010: User Guide. US Environmental Protection Agency (EPA). 150 p. Available from Internet: http://www.epa.gov/oms/ models/moves/420b09041.pdf.

Fajardo, D.; Au, T.-C.; Waller, S.T.; Stone, P.; Yang, D. 2011. Automated intersection control: performance of future innovation versus current traffic signal control, Transportation Research Record: Journal of the Transportation Research Board 2259: 223-232. http://dx.doi.org/10.3141/2259-21

Frey, H. C.; Rouphail, N. M.; Zhai, H. 2006. Speed- and facilityspecific emission estimates for on-road light-duty vehicles on the basis of real-world speed profiles, Transportation Research Record: Journal of the Transportation Research Board 1987: 128-137. http://dx.doi.org/10.3141/1987-14

HCM. 2010. Highway Capacity Manual. Transportation Research Board. 5th edition. $1650 \mathrm{p}$.

HCS. 2010. Highway Capacity Software.

Hu, B.; Gharavi, H. 2011. A joint vehicle-vehicle/vehicle-roadside communication protocol for highway traffic safety, International Journal of Vehicular Technology 2011: 1-10. http://dx.doi.org/10.1155/2011/718048

Jiménez-Palacios, J. L. 1999. Understanding and Quantifying Motor Vehicle Emissions with Vehicle Specific Power and TILDAS Remote Sensing: PhD Dissertation. Massachusetts Institute of Technology. 361 p. Available from Internet: http://dspace.mit.edu/handle/1721.1/44505.

Li, Z.; Chitturi, M. V.; Noyce, D. A. 2013a. Development of Next Generation Intersection Control. Final Report CFIRE 0418. University of Wisconsin-Madison, US. 84 p. Available from Internet: http://www.wistrans.org/cfire/documents/ FR_0418.pdf .

Li, Z.; Chitturi, M. V.; Zheng, D.; Bill, A. R.; Noyce, D. A. 2013b. Modeling reservation-based autonomous intersection control in VISSIM, Transportation Research Record: Journal of the Transportation Research Board 2381: 81-90. http://dx.doi.org/10.3141/2381-10 
Li, Z.; Chitturi, M. V.; Zheng, D.; Bill, A. R.; Noyce, D. A. 2013c. Next-generation intersection control algorithm for autonomous vehicles, in TRB 92nd Annual Meeting Compendium of Papers, 13-17 January 2013, Washington, DC, $1-21$.

Mahmod, M.; Van Arem, B.; Pueboobpaphan, R.; De Lange, R. 2013. Reducing local traffic emissions at urban intersection using ITS countermeasures, IET Intelligent Transport Systems 7(1): 78-86.

http://dx.doi.org/10.1049/iet-its.2011.0222

NHTSA. 2013. Traffic Crash Statistics. National Highway Traffic Safety Administration (NHTSA). Available from Internet: http://www.nhtsa.gov.

Pandian, S.; Gokhale, S.; Ghoshal, A. K. 2009. Evaluating effects of traffic and vehicle characteristics on vehicular emissions near traffic intersections, Transportation Research Part D: Transport and Environment 14(3): 180-196. http://dx.doi.org/10.1016/j.trd.2008.12.001

Quinlan, M.; Au, T.-C.; Zhu, J.; Stiurca, N.; Stone, P. 2010. Bringing simulation to life: a mixed reality autonomous intersection, in The IEEE/RSJ 2010 International Conference on Intelligent Robots and Systems (IROS): Conference Proceedings, 18-22 October 2010, Taipei, Taiwan, 6083-6088. http://dx.doi.org/10.1109/IROS.2010.5651993

Shahidi, N.; Au, T.-C.; Stone, P. 2011. Batch reservations in autonomous intersection management, in AAMAS 2011 the 10th International Conference on Autonomous Agents and Multiagent Systems: Proceedings, 2-6 May 2011, Taipei, Taiwan, 3: 1225-1226.

VanMiddlesworth, M.; Dresner, K.; Stone, P. 2008. Replacing the stop sign: unmanaged intersection control for autonomous vehicles, in AAMAS'08: Proceedings of the 7th International Joint Conference on Autonomous Agents and Multiagent Systems, 12-16 May 2008, Estoril, Portugal, 3: 1413-1416.

Vasirani, M.; Ossowski, S. 2009. Evaluating policies for reservation-based intersection control, in Progress in Artificial Intelligence: 14th Portuguese Conference on Artificial Intelligence, EPIA 2009, 12-15 October 2009, Aveiro, Portugal.

Wikipedia. 2014a. Autonomous Car. Available from Internet: https://en.wikipedia.org/wiki/Autonomous_car

Wikipedia. 2014b. Google Driverless Car. Available from Internet: https://en.wikipedia.org/wiki/Google_driverless_car

Wu, J.; Abbas-Turki, A.; Correia, A.; El Moudni, A. 2007. Discrete intersection signal control, in IEEE International Conference on Service Operations and Logistics, and Informatics, 2007: SOLI 2007, 27-29 August 2007, Philadelphia, PA, USA, 1-6. http://dx.doi.org/10.1109/SOLI.2007.4383891

Wu, J.; Abbas-Turki, A.; El Moudni, A. 2010. Contextualized traffic controlling at isolated urban intersection, in The 14th World Multi-Conference on Systemics, Cybernetics and Informatics: WMSCI 2010, 29 June 29-2 July 2010, Orlando, USA, 1-6. Available from Internet: http://www.iiis.org/ CDs2010/CD2010SCI/SCI_2010/PapersPdf/SA803SV.pdf

Yan, F.; Dridi, M.; El Moudni, A. 2009. Autonomous vehicle sequencing algorithm at isolated intersections, in 12th International IEEE Conference on Intelligent Transportation Systems, 2009: ITSC'09, 4-7 October 2009, St. Louis, MO. http://dx.doi.org/10.1109/ITSC.2009.5309708
Yan, F.; Dridi, M.; El Moudni, A. 2008. Control of traffic lights in intersection: a new branch and bound approach, in 2008 International Conference on Service Systems and Service Management: Proceedings of ICSSSM'08, 30 June - 2 July 2008, Melbourne, Australia, 1-6. http://dx.doi.org/10.1109/ICSSSM.2008.4598496

Yao, Z.; Wei, H.; Li, Z.; Ma, T.; Liu, H.; Yang, Y. J. 2013. Developing operating mode distribution inputs for moves with a computer vision-based vehicle data collector, Transportation Research Record: Journal of the Transportation Research Board 2340: 49-58. http://dx.doi.org/10.3141/2340-06

Yu, L.; Jia, S.; Shi, Q. 2009. Research on transportation-related emissions: current status and future directions, Journal of the Air \& Waste Management Association 59(2):183-195. http://dx.doi.org/10.3155/1047-3289.59.2.183 